Proyecciones Journal of Mathematics

Vol. 31, No 4, pp. 309-332, December 2012.

Universidad Católica del Norte

Antofagasta - Chile

\title{
On some refinements of companions of Fejér's inequality via superquadratic functions
}

\author{
Muhammad Amer Latif \\ University of Hail, Saudi Arabia \\ Received : December 2011. Accepted : September 2012
}

\begin{abstract}
In this paper some companions of Fejér's inequality for superquadratic functions are given, we also get refinements of some known results proved in [18].
\end{abstract}

Subjclass : [2000] 26D15.

Keywords : Hermite-Hadamard Inequality, convex function, Fejér inequality, superquadratic function. 


\section{Introduction}

Let $\emptyset \neq I \subseteq R, a, b \in I$ with $a<b$, let $f: I \rightarrow R$ be a convex function and $p:[a, b] \rightarrow R$ be a non-negative integrable and symmetric about $x=\frac{a+b}{2}$. The following two inequalities are of great significance in literature: the first known as Hermite-Hadamard inequality:

$$
f\left(\frac{a+b}{2}\right) \leq \frac{1}{b-a} \int_{a}^{b} f(x) d x \leq \frac{f(a)+f(b)}{2}
$$

with the reversed inequality for the concave function $f$, and the second, known as Fejér's inequality:

$$
f\left(\frac{a+b}{2}\right) \int_{a}^{b} p(x) d x \leq \int_{a}^{b} f(x) p(x) d x \leq \frac{f(a)+f(b)}{2} \int_{a}^{b} p(x) d x .
$$

These inequalities attracted the attention of many mathematicians over the decades and they generalize, improve and extend these inequalities in a number of ways, see $[6,7,8,9,11,19]$. Let us now define some mappings and quote the results established by K.L. Tseng, S. R. Hwang and S.S. Dragomir in [18]:

$$
\begin{gathered}
G(t)=\frac{1}{2}\left[f\left(t a+(1-t) \frac{a+b}{2}\right)+f\left(t b+(1-t) \frac{a+b}{2}\right)\right], \\
H(t)=\frac{1}{b-a} \int_{a}^{b} f\left(t x+(1-t) \frac{a+b}{2}\right) d x \\
I(t)=\int_{a}^{b} \frac{1}{2}\left[f\left(t \frac{x+a}{2}+(1-t) \frac{a+b}{2}\right)\right. \\
\left.+f\left(t \frac{x+b}{2}+(1-t) \frac{a+b}{2}\right)\right] p(x) d x, \\
L_{p}(t)=\frac{1}{2} \int_{a}^{b}[f(t a+(1-t) x)+f(t b+(1-t) x)] p(x) d x, \\
L_{p}(t)=\frac{1}{2(b-a)} \int_{a}^{b}[f(t a+(1-t) x)+f(t b+(1-t) x)] d x
\end{gathered}
$$


and

$$
\begin{gathered}
S_{p}(t)=\frac{1}{4} \int_{a}^{b}\left[f\left(t a+(1-t) \frac{x+a}{2}\right)+f\left(t a+(1-t) \frac{x+b}{2}\right)\right. \\
\left.+f\left(t b+(1-t) \frac{x+a}{2}\right)+f\left(t b+(1-t) \frac{x+b}{2}\right)\right] p(x) d x,
\end{gathered}
$$

where $f:[a, b] \rightarrow R$ is a convex function and $p:[a, b] \rightarrow R$ is non-negative integrable and symmetric about $x=\frac{a+b}{2}, t \in[0,1]$.

Now we quote some results from [18]:

Theorem 1. [18] Let $f, p, I$ be defined as above. Then:

1. The following inequality holds:

$$
\begin{gathered}
f\left(\frac{a+b}{2}\right) \int_{a}^{b} p(x) d x \\
\leq 2\left[\int_{\frac{3 a+b}{4}}^{\frac{a+b}{2}} f(x) p(4 x-2 a-b) d x+\int_{\frac{a+b}{2}}^{\frac{a+3 b}{4}} f(x) p(4 x-a-2 b) d x\right] \\
\leq \int_{0}^{1} I(t) d t \leq \frac{1}{2}\left[f\left(\frac{a+b}{2}\right) \int_{a}^{b} p(x) d x\right. \\
\left.+\int_{a}^{b} \frac{1}{2}\left[f\left(\frac{x+a}{2}\right)+f\left(\frac{x+b}{2}\right)\right] p(x) d x\right] .
\end{gathered}
$$

2. If $f$ is differentiable on $[a, b]$ and $p$ is bounded on $[a, b]$, then for all $t \in[0,1]$ we have the inequality

$$
0 \leq \int_{a}^{b} \frac{1}{2}\left[f\left(\frac{x+a}{2}\right)+f\left(\frac{x+b}{2}\right)\right] p(x) d x-I(t)
$$

$$
\leq(1-t)\left[\frac{f(a)+f(b)}{2}(b-a)-\int_{a}^{b} f(x) d x\right]\|p\|_{\infty},
$$

where $\|p\|_{\infty}=\sup _{x \in[a, b]}|p(x)|$. 
3. If $f$ is differentiable on $[a, b]$, then for all $t \in[0,1]$ we have the inequality

$0 \leq \frac{f(a)+f(b)}{2} \int_{a}^{b} p(x) d x-I(t) \leq \frac{\left(f^{\prime}(b)-f^{\prime}(a)\right)(b-a)}{4} \int_{a}^{b} p(x) d x$.

Theorem 2. [18] Let $f, p, G, I$ be defined as above. Then:

1. The following inequality holds for all $t \in[0,1]$ :

$$
I(t) \leq G(t) \int_{a}^{b} p(x) d x .
$$

2. If $f$ is differentiable on $[a, b]$ and $p$ is bounded on $[a, b]$, then for all $t \in[0,1]$ we have the inequality

$$
0 \leq I(t)-f\left(\frac{a+b}{2}\right) \int_{a}^{b} p(x) d x \leq(b-a)[G(t)-H(t)]\|p\|_{\infty}
$$

where $\|p\|_{\infty}=\sup _{x \in[a, b]}|p(x)|$.

Theorem 3. [18] Let f, $p, G, I, S_{p}$ be defined as above. Then we have the following results:

1. $S_{p}$ is convex on $[0,1]$.

2. The following inequalities hold for all $t \in[0,1]$ :

$$
\begin{gathered}
G(t) \int_{a}^{b} p(x) d x \leq S_{p}(t) \\
\leq(1-t) \int_{a}^{b} \frac{1}{2}\left[f\left(\frac{x+a}{2}\right)+f\left(\frac{x+b}{2}\right)\right] p(x) d x
\end{gathered}
$$




$$
+t \cdot \frac{f(a)+f(b)}{2} \int_{a}^{b} p(x) d x \leq \frac{f(a)+f(b)}{2} \int_{a}^{b} p(x) d x,
$$

and

$$
I(1-t) \leq S_{p}(t)
$$

3. The following equality holds:

$$
\sup _{x \in[0,1]} S_{p}(t)=S_{p}(1)=\frac{f(a)+f(b)}{2} \int_{a}^{b} p(x) d x .
$$

They used the following Lemma to prove the above results:

Lemma 4. $[17, p .3] f:[a, b] \longrightarrow R$ be convex function and let $a \leq A \leq$ $C \leq D \leq B \leq b$ with $A+B=C+D$. Then

$$
f(A)+f(B) \leq f(C)+f(D) .
$$

Let us now recall the definition, some of the properties and results related to superquadratic functions to be used in the sequel.

Definition 5. [3, Defintion 2.1] Let $I=[0, a]$ or $[0, \infty)$ be an interval in $R$. A function $f: I \longrightarrow R$ is superquadratic if for each $x$ in $I$ there exists a real number $C(x)$ such that

$$
f(y)-f(x) \geq C(x)(y-x)+f(|y-x|)
$$

for all $y \in I$. If $-f$ is superquadratic then $f$ is called subquadratic.

For examples of superquadratic functions see [2, p. 1049].

Theorem 6. [3, Theorem 2.3] The inequality

$$
f\left(\int g d \mu\right) \leq \int\left(f(g(s))-f\left(\left|g(s)-\int g d \mu\right|\right)\right) d \mu(s)
$$

holds for all probability measure $\mu$ and all non-negative $\mu$-integrable function $g$, if and only if $f$ is superquadratic. 
The following discrete version of the above theorem will be helpful in the sequel of the paper:

Lemma 7. [2, Lemma A, p.1049] Suppose that $f$ is superquadratic. Let $x_{r} \geq 0,1 \leq r \leq n$, and let $\bar{x}=\sum_{r=1}^{n} \lambda_{r} x_{r}$ where $\lambda_{r} \geq 0$ and $\sum_{r=1}^{n} \lambda_{r}=1$. Then

$$
\sum_{r=1}^{n} \lambda_{r} f\left(x_{r}\right) \geq f(\bar{x})+\sum_{r=1}^{n} \lambda_{r} f\left(\left|x_{r}-\bar{x}\right|\right) \text {. }
$$

The following Lemma shows that positive superquadratic functions are also convex:

Lemma 8. [3, Lemma 2.2] Let $f$ be superquadratic function with $C(x)$ as in Definition 1. Then

1. $f(0) \leq 0$.

2. If $f(0)=f^{\prime}(0)=0$ then $C(x)=f^{\prime}(x)$ whenever $f$ is differentiable at $x>0$.

3. If $f \geq 0$, then $f$ convex and $f(0)=f^{\prime}(0)=0$.

In [4] a converse of Jensen's inequality for superquadratic functions was proved:

Theorem 9. [4, Theorem 1] Let $(\Omega, A, \mu)$ be a measurable space with $0<$ $\mu(\Omega)<\infty$ and let $f:[0, \infty) \rightarrow R$ be a superquadratic function. If $g$ : $\Omega \rightarrow[m, M] \subseteq[0, \infty)$ is such that $g, f \circ g \in L_{1}(\mu)$, then we have for $\bar{g}=\frac{1}{\mu(\Omega)} \int g d \mu$,

$$
\begin{gathered}
\frac{1}{\mu(\Omega)} \int f(g) d \mu \leq \frac{M-\bar{g}}{M-m} f(m)+\frac{\bar{g}-m}{M-m} f(M) \\
-\frac{1}{\mu(\Omega)} \frac{1}{M-m} \int((M-g) f(g-m)+(g-m) f(M-g)) d \mu .
\end{gathered}
$$

The discrete version of this theorem is:

Theorem 10. [4, Theorem 2] Let $f:[0, \infty) \rightarrow R$ be a superquadratic function. Let $\left(x_{1}, \ldots, x_{n}\right)$ be an $n$-tuple in $[m, M]^{n}(0 \leq m \leq M<\infty)$, and $\left(p_{1}, \ldots, p_{n}\right)$ be a non-negative $n$-tuple such that $P_{n}=\sum_{i=1}^{n} p_{i}>0$. Denote $\bar{x}=\frac{1}{P_{n}} \sum_{i=1}^{n} p_{i} x_{i}$, then 


$$
\begin{gathered}
\frac{1}{P_{n}} \sum_{i=1}^{n} p_{i} f\left(x_{i}\right) \leq \frac{M-\bar{x}}{M-m} f(m)+\frac{\bar{x}-m}{M-m} f(M) \\
-\frac{1}{P_{n}(M-m)} \sum_{i=1}^{n} p_{i}\left[\left(M-x_{i}\right) f\left(x_{i}-m\right)+\left(x_{i}-m\right) f\left(M-x_{i}\right)\right]
\end{gathered}
$$

For recent results on Fejér and Hermite-Hadamard type inequalities for superquadratic functions, we refer interested readers to [4], [5] and [2]. In this paper we deal with mappings $G(t), I(t), S_{p}(t)$ and $L(t)$ when $f$ is superquadratic function. In case when superquadratic function $f$ is also non-negative and hence convex we get refinements of some parts of Theorem 1, Theorem 2 and of Theorem 3.

\section{Main Results}

In this section we prove our main results by using the same techniques as used in [17] and [2]. Moreover, we assume that all the considered integrals in this section exist.

In order to prove our main results we go through some calculations. From Lemma 2 and Theorem 6 for $n=2$, we get that

$f(z) \leq \frac{M-z}{M-m} f(m)+\frac{z-m}{M-m} f(M)-\frac{M-z}{M-m} f(z-m)-\frac{z-m}{M-m} f(M-z)$

and

$f(M+m-z) \leq \frac{z-m}{M-m} f(m)+\frac{M-z}{M-m} f(M)-\frac{z-m}{M-m} f(M-z)-\frac{M-z}{M-m} f(z-m)$

hold for superquadratic function $f, 0 \leq m \leq z \leq M, m<M$.

Therefore from (2.1) and (2.2), we have

$f(z)+f(M+m-z) \leq f(m)+f(M)-2 \frac{z-m}{M-m} f(M-z)-2 \frac{M-z}{M-m} f(z-m)$.

Now for $0 \leq t \leq \frac{1}{2}$ and $0 \leq a \leq x \leq \frac{a+b}{2}$, we obtain from (2.3) the following inequalities: 
By setting $z=\frac{a+b}{2}, M=\frac{3(a+b)}{4}-\frac{x}{2}, m=\frac{x}{2}+\frac{a+b}{4}$ in (2.3), we have that $2 f\left(\frac{a+b}{2}\right) \leq f\left(\frac{x}{2}+\frac{a+b}{4}\right)+f\left(\frac{3(a+b)}{4}-\frac{x}{2}\right)-2 f\left(\frac{1}{2}\left(\frac{a+b}{2}-x\right)\right)$

holds.

Also, by replacing $z=\frac{x}{2}+\frac{a+b}{4}, M=t x+(1-t) \frac{a+b}{2}, m=t \frac{a+b}{2}+(1-t) x$ in (2.3), we get that

$$
\begin{aligned}
2 f\left(\frac{x}{2}+\frac{a+b}{4}\right) \leq & f\left(t \frac{a+b}{2}+(1-t) x\right)+f\left(t x+(1-t) \frac{a+b}{2}\right) \\
& -2 f\left(\left(\frac{1}{2}-t\right)\left(\frac{a+b}{2}-x\right)\right)
\end{aligned}
$$

holds.

Further, for $z=\frac{3(a+b)}{4}-\frac{x}{2}, M=t \frac{a+b}{2}+(1-t)(a+b-x)$, $m=t(a+b-x)+(1-t) \frac{a+b}{2}$ in $(2.3)$, we observe that

$$
\begin{gathered}
2 f\left(\frac{3(a+b)}{4}-\frac{x}{2}\right) \leq f\left(t(a+b-x)+(1-t) \frac{a+b}{2}\right) \\
+f\left(t \frac{a+b}{2}+(1-t)(a+b-x)\right)-2 f\left(\left(\frac{1}{2}-t\right)\left(\frac{a+b}{2}-x\right)\right)
\end{gathered}
$$

holds.

Again, for $z=t \frac{a+b}{2}+(1-t) x, M=\frac{a+b}{2}, m=x$ in (2.3), we observe that

$$
f\left(t \frac{a+b}{2}+(1-t) x\right)+f\left(t x+(1-t) \frac{a+b}{2}\right)
$$

$\leq f(x)+f\left(\frac{a+b}{2}\right)-2 t f\left((1-t)\left(\frac{a+b}{2}-x\right)\right)-2(1-t) f\left(t\left(\frac{a+b}{2}-x\right)\right)$

holds.

Finally, by setting $z=t(a+b-x)+(1-t) \frac{a+b}{2}, M=a+b-x, m=\frac{a+b}{2}$ in (2.3), we get that 


$$
\begin{gathered}
f\left(t(a+b-x)+(1-t) \frac{a+b}{2}\right)+f\left(t \frac{a+b}{2}+(1-t)(a+b-x)\right) \\
(2.8) \leq f\left(\frac{a+b}{2}\right)+f(a+b-x)-2 t f\left((1-t)\left(\frac{a+b}{2}-x\right)\right) \\
-2(1-t) f\left(t\left(\frac{a+b}{2}-x\right)\right)
\end{gathered}
$$

holds.

Now we are ready to state and prove our main results based on the calculations done above.

Theorem 1. Let $f$ be superquadratic integrable function on $[0, b]$ and $p(x)$ be non-negative integrable and symmetric about $x=\frac{a+b}{2}, 0 \leq a<b$. Let $I$ be defined as above, then we have the following inequalities:

$$
\begin{aligned}
& f\left(\frac{a+b}{2}\right) \int_{a}^{b} p(x) d x \leq 2\left[\int_{\frac{3 a+b}{4}}^{\frac{a+b}{2}} f(x) p(4 x-2 a-b) d x\right. \\
& \left.+\int_{\frac{a+b}{2}}^{\frac{a+3 b}{4}} f(x) p(4 x-a-2 b) d x\right]-\int_{a}^{b} f\left(\frac{1}{4}(b-x)\right) p(x) d x \\
& \text { (2.10) } 2\left[\int_{\frac{3 a+b}{4}}^{\frac{a+b}{2}} f(x) p(4 x-2 a-b) d x+\int_{\frac{a+b}{2}}^{\frac{a+3 b}{4}} f(x) p(4 x-a-2 b) d x\right] \\
& \leq \int_{0}^{1} I(t) d t-\int_{a}^{b} \int_{0}^{1} f\left(\left|\frac{1}{2}-t\right|\left(\frac{b-x}{2}\right)\right) p(x) d t d x
\end{aligned}
$$

and

$$
\begin{gathered}
\int_{0}^{1} I(t) d t \leq \frac{1}{2}\left[f\left(\frac{a+b}{2}\right) \int_{a}^{b} p(x) d x+\int_{a}^{b} \frac{1}{2}\left[f\left(\frac{x+a}{2}\right)\right.\right. \\
\left.\left.+f\left(\frac{x+b}{2}\right)\right] p(x) d x\right]-2 \int_{a}^{b} \int_{0}^{1}(1-t) f\left(t \frac{b-x}{2}\right) p(x) d t d x
\end{gathered}
$$


Proof. Using simple techniques of integration and by the assumptions on $p$, we have

$$
f\left(\frac{a+b}{2}\right) \int_{a}^{b} p(x) d x=4 \int_{a}^{\frac{a+b}{2}} \int_{0}^{\frac{1}{2}} f\left(\frac{a+b}{2}\right) p(2 x-a) d t d x .
$$

Therefore from (2.4), we get that

$$
\begin{gathered}
f\left(\frac{a+b}{2}\right) \int_{a}^{b} p(x) d x \\
\leq 2 \int_{a}^{\frac{a+b}{2}} \int_{0}^{\frac{1}{2}}\left[f\left(\frac{x}{2}+\frac{a+b}{4}\right)+f\left(\frac{3(a+b)}{4}-\frac{x}{2}\right)\right] p(2 x-a) d t d x \\
-4 \int_{a}^{\frac{a+b}{2}} \int_{0}^{\frac{1}{2}} f\left(\frac{1}{2}\left(\frac{a+b}{2}-x\right)\right) p(2 x-a) d t d x
\end{gathered}
$$

But

$$
2 \int_{a}^{\frac{a+b}{2}} \int_{0}^{\frac{1}{2}}\left[f\left(\frac{x}{2}+\frac{a+b}{4}\right)+f\left(\frac{3(a+b)}{4}-\frac{x}{2}\right)\right] p(2 x-a) d t d x
$$

$$
\begin{gathered}
=2 \int_{\frac{3 a+b}{4}}^{\frac{a+b}{2}}[f(x)+f(a+b-x)] p(4 x-2 a-b) d x \\
=2\left[\int_{\frac{3 a+b}{4}}^{\frac{a+b}{2}} f(x) p(4 x-2 a-b) d x+\int_{\frac{a+b}{2}}^{\frac{a+3 b}{4}} f(x) p(4 x-a-2 b) d x\right] .
\end{gathered}
$$

From (2.12), (2.13) and by the change of variable $x \rightarrow \frac{x+a}{2}$, we get (2.9). From (2.5), (2.6) and (2.13), we have

$$
2\left[\int_{\frac{3 a+b}{4}}^{\frac{a+b}{2}} f(x) p(4 x-2 a-b) d x+\int_{\frac{a+b}{2}}^{\frac{a+3 b}{4}} f(x) p(4 x-a-2 b) d x\right]
$$




$$
\begin{aligned}
& \quad \leq \int_{a}^{\frac{a+b}{2}} \int_{0}^{\frac{1}{2}}\left[f\left(t \frac{a+b}{2}+(1-t) x\right)+f\left(t x+(1-t) \frac{a+b}{2}\right)\right. \\
& +\mathrm{f}\left(t(a+b-x)+(1-t) \frac{a+b}{2}\right) \\
& \left.+f\left(t \frac{a+b}{2}+(1-t)(a+b-x)\right)\right] p(2 x-a) d t d x \\
& -4 \int_{a}^{\frac{a+b}{2}} \int_{0}^{\frac{1}{2}} f\left(\left(\frac{1}{2}-t\right)\left(\frac{a+b}{2}-x\right)\right) p(2 x-a) d t d x .
\end{aligned}
$$

But

$$
\int_{0}^{1} I(t) d t=\int_{a}^{\frac{a+b}{2}} \int_{0}^{\frac{1}{2}}\left[f\left(t \frac{a+b}{2}+(1-t) x\right)+f\left(t x+(1-t) \frac{a+b}{2}\right)\right.
$$

$$
+f\left(t(a+b-x)+(1-t) \frac{a+b}{2}\right)
$$

$\left.+f\left(t \frac{a+b}{2}+(1-t)(a+b-x)\right)\right] p(2 x-a) d t d x$.

From (2.14) and (2.15), we get that

$$
2\left[\int_{\frac{3 a+b}{4}}^{\frac{a+3 b}{4}} f(x) p(4 x-2 a-b) d x+\int_{\frac{3 a+b}{4}}^{\frac{a+3 b}{4}} f(x) p(4 x-a-2 b) d x\right]
$$

$$
\leq \int_{0}^{1} I(t) d t-4 \int_{a}^{\frac{a+b}{2}} \int_{0}^{\frac{1}{2}} f\left(\left(\frac{1}{2}-t\right)\left(\frac{a+b}{2}-x\right)\right) p(2 x-a) d t d x .
$$

By the change of variables $t \rightarrow 1-t$ and $x \rightarrow \frac{x+a}{2}$ in (2.16), we get

From (2.7), (2.8) and (2.15), we have

$$
\int_{0}^{1} I(t) d t \leq \int_{0}^{\frac{1}{2}} \int_{a}^{\frac{a+b}{2}}\left[f(x)+2 f\left(\frac{a+b}{2}\right)-4 t f\left((1-t)\left(\frac{a+b}{2}-x\right)\right)\right.
$$


$\left.-4(1-t) f\left(t\left(\frac{a+b}{2}-x\right)\right)+f(a+b-x)\right] p(2 x-a) d t d x$.

But

$$
\frac{1}{2}\left[f\left(\frac{a+b}{2}\right) \int_{a}^{b} p(x) d x+\int_{a}^{b} \frac{1}{2}\left[f\left(\frac{x+a}{2}\right)+f\left(\frac{x+b}{2}\right)\right] p(x) d x\right]
$$

$$
=\int_{a}^{\frac{a+b}{2}} \int_{0}^{\frac{1}{2}}\left[f(x)+2 f\left(\frac{a+b}{2}\right)+f(a+b-x)\right] p(2 x-a) d t d x .
$$

From $(2.17),(2.18)$ and by the change of variables $x \rightarrow \frac{x+a}{2}$ and $t \rightarrow$ $1-t$, we get (2.11).

This completes the proof of the theorem as well.

Remark 2. If the superquadratic function $f$ is non-negative and hence convex, then from (2.9) we get refinement of the first inequality of (1.3) in Theorem 1; from (2.10) we get refinement of the middle inequality of (1.3) in Theorem 1 and from (2.11) we get refinement of the last inequality of (1.3) in Theorem 1.

Corollary 3. Let $f$ be superquadratic integrable function on $[0, b]$. If $p(x)=\frac{1}{b-a}, x \in[a, b]$ and $0 \leq a<b$, then we have

$$
f\left(\frac{a+b}{2}\right) \leq \frac{2}{b-a} \int_{\frac{3 a+b}{4}}^{\frac{a+3 b}{4}} f(x) d x-\frac{1}{b-a} \int_{a}^{b} f\left(\frac{1}{4}(b-x)\right) d x
$$

$\frac{2}{b-a} \int_{\frac{3 a+b}{4}}^{\frac{a+3 b}{4}} f(x) d x \leq \int_{0}^{1} H(t) d t-\frac{1}{b-a} \int_{a}^{b} \int_{0}^{1} f\left(\left|\frac{1}{2}-t\right|\left(\frac{b-x}{2}\right)\right) d t d x$

and

$$
\begin{gathered}
\int_{0}^{1} H(t) d t \leq \frac{1}{2}\left[f\left(\frac{a+b}{2}\right)+\frac{1}{b-a} \int_{a}^{b} f(x) d x\right] \\
-\frac{2}{b-a} \int_{a}^{b} \int_{0}^{1}(1-t) f\left(t \frac{b-x}{2}\right) d t d x
\end{gathered}
$$

where

$$
H(t)=\frac{1}{b-a} \int_{a}^{b} f\left(t x+(1-t) \frac{a+b}{2}\right) d x, t \in[0,1] .
$$


Proof. If $p(x)=\frac{1}{b-a}, x \in[a, b]$, then $I(t)=H(t), t \in[0,1]$, and therefore the proof of the corollary follows directly from the above theorem.

Remark 4. If the superquadratic function $f$ is non-negative and therefore convex, then the inequalities in Corollary 1 refine the inequalities in (1.3) of Theorem $B$ from $[18, p .2]$.

To proceed to our next result, we go again through the similar calculations as given before Theorem 7 .

For $0 \leq a \leq x \leq \frac{a+b}{2}, t \in[0,1]$, we have that

$$
\begin{aligned}
a \leq t a+(1-t) \frac{a+b}{2} \leq & t x+(1-t) \frac{a+b}{2} \leq t(a+b-x)+(1-t) \frac{a+b}{2} \\
& \leq t b+(1-t) \frac{a+b}{2} \leq b .
\end{aligned}
$$

Therefore, by replacing $z=t x+(1-t) \frac{a+b}{2}, M=t b+(1-t) \frac{a+b}{2}, m=$ $t a+(1-t) \frac{a+b}{2}$ in $(2.3)$,

we get that

$$
\begin{gathered}
f\left(t(a+b-x)+(1-t) \frac{a+b}{2}\right)+f\left(t x+(1-t) \frac{a+b}{2}\right) \\
\leq f\left(t b+(1-t) \frac{a+b}{2}\right)+f\left(t a+(1-t) \frac{a+b}{2}\right) \\
\quad-\frac{2(x-a)}{b-a} f(t(b-x))-\frac{2(b-x)}{b-a} f(t(x-a))
\end{gathered}
$$

holds.

Now we are ready to state and prove our next result based on the above calculations.

Theorem 5. Let $f$ be superquadratic integrable function on $[0, b]$ and $p(x)$ be non-negative integrable and symmetric about $x=\frac{a+b}{2}, 0 \leq a<b$. Let $I$ and $G$ be defined as above, then the following inequality holds for all $t \in[0,1]:$

$$
\begin{gathered}
I(t) \leq G(t) \int_{a}^{b} p(x) d x-\int_{a}^{b} \frac{1}{2}\left[\frac{x-a}{b-a} f\left(t\left(\frac{2 b-x-a}{2}\right)\right)\right. \\
\left.+\frac{2 b-x-a}{b-a} f\left(t\left(\frac{x-a}{2}\right)\right)\right] p(x) d x
\end{gathered}
$$


Proof. Using simple techniques of integration and by the assumptions on $p$, we have that the following identity holds for all $t \in[0,1]$ :

$$
\begin{gathered}
G(t) \int_{a}^{b} p(x) d x=\int_{a}^{\frac{a+b}{2}}\left[f\left(t a+(1-t) \frac{a+b}{2}\right)\right. \\
\left.+f\left(t b+(1-t) \frac{a+b}{2}\right)\right] p(2 x-a) d x .
\end{gathered}
$$

Arguing similarly as in obtaining (2.15), by using (2.22) and (2.24), we get that

$$
I(t) \leq G(t) \int_{a}^{b} p(x) d x
$$

$$
-\int_{a}^{\frac{a+b}{2}}\left[\frac{2(x-a)}{b-a} f(t(b-x))+\frac{2(b-x)}{b-a} f(t(x-a))\right] p(2 x-a) d x,
$$

for all $\in[0,1]$.

By the change of variable $x \rightarrow \frac{x+a}{2}$ in (2.25), we get (2.23).

This completes the proof of the theorem.

Remark 6. If the superquadratic function $f$ is non-negative and hence convex, then the inequality (2.23) represents a refinement of the inequality (1.6) in Theorem 2.

Corollary 7. Let $f$ be superquadratic integrable function on $[0, b]$, let $p(x)=\frac{1}{b-a}, 0 \leq a<b$ and $G, H$ be defined as above. Then for all $t \in[0,1]$, we have the following inequality

$$
\begin{gathered}
H(t) \leq G(t)-\int_{a}^{b} \frac{1}{2(b-a)}\left[\frac{x-a}{b-a} f\left(t\left(\frac{2 b-x-a}{2}\right)\right)\right. \\
\left.+\frac{2 b-x-a}{b-a} f\left(t\left(\frac{x-a}{2}\right)\right)\right] d x .
\end{gathered}
$$

Proof. This is a direct consequence of the above theorem, since for $p(x)=\frac{1}{b-a}, x \in[a, b], I(t)=H(t)$, for all $t \in[0,1]$.

Remark 8. If the superquadratic function $f$ is non-negative and hence convex, then the inequality (2.26) represents refinement of the inequality (1.6) in [18, Theorem C, p. 2]. 
Now again we give some calculations for our next result.

For $0 \leq t \leq \frac{1}{2}$ and $0 \leq a \leq x \leq \frac{a+b}{2}$, we obtain from (2.3) the following inequalities:

By setting $m=t a+(1-t) x, M=t a+(1-t)(a+b-x)$ and $z=$ $t a+(1-t) \frac{a+b}{2}$ in $(2.3)$, we obseve that

$$
2 f\left(t a+(1-t) \frac{a+b}{2}\right) \leq f(t a+(1-t) x)+f(t a+(1-t)(a+b-x))
$$

$$
-2 f\left((1-t)\left(\frac{a+b}{2}-x\right)\right)
$$

holds.

Also, by replacing $m=t b+(1-t) x, M=t b+(1-t)(a+b-x)$ and $z=t b+(1-t) \frac{a+b}{2}$ in $(2.3)$, we get that

$$
2 f\left(t b+(1-t) \frac{a+b}{2}\right) \leq f(t b+(1-t) x)+f(t b+(1-t)(a+b-x))
$$

$$
-2 f\left((1-t)\left(\frac{a+b}{2}-x\right)\right)
$$

holds.

Theorem 9. Let $f$ be superquadratic integrable function on $[0, b]$ and $p(x)$ be non-negative integrable and symmetric about $x=\frac{a+b}{2}, 0 \leq a<b$. Let $S_{p}$ and $G$ be defined as above, then the following inequality holds for all $t \in[0,1]:$

$$
G(t) \int_{a}^{b} p(x) d x \leq S_{p}(t)-\int_{a}^{b} f\left((1-t)\left(\frac{b-x}{2}\right)\right) p(x) d x
$$

Proof. By the simple techniques of integration and by the assumptions on $p$, we have the following identity for all $t \in[0,1]$ :

$$
\begin{aligned}
& S_{p}(t)=\frac{1}{2} \int_{a}^{\frac{a+b}{2}}[f(t a+(1-t) x)+f(t a+(1-t)(a+b-x)) \\
& +f(t b+(1-t) x)+f(t b+(1-t)(a+b-x))] p(2 x-a) d x
\end{aligned}
$$


From (2.27), (2.28) and (2.30), we have that

$$
\int_{a}^{\frac{a+b}{2}}\left[f\left(t a+(1-t) \frac{a+b}{2}\right)+f\left(t b+(1-t) \frac{a+b}{2}\right)\right] p(2 x-a) d x
$$

$$
\leq S_{p}(t)-2 \int_{a}^{\frac{a+b}{2}} f\left((1-t)\left(\frac{a+b}{2}-x\right)\right) p(2 x-a) d x,
$$

holds for all $t \in[0,1]$.

From (2.24) and by the change of variable $x \rightarrow \frac{a+x}{2}$, we get from (2.31) that

$$
G(t) \int_{a}^{b} p(x) d x \leq S_{p}(t)-\int_{a}^{b} f\left((1-t)\left(\frac{b-x}{2}\right)\right) p(x) d x,
$$

for all $t \in[0,1]$. Which is (2.29) and this completes the proof of the theorem as well.

Remark 10. The result of the above theorem refines the first inequality of Theorem 3, when superquadratic function $f$ is non-negative and hence convex.

Corollary 11. Let $f$ be superquadratic integrable function on $[0, b]$ and let $p(x)=\frac{1}{b-a}, x \in[a, b], 0 \leq a<b$. Let $G$ be defined as above, then the following inequality holds for all $t \in[0,1]$ :

$$
G(t) \leq L(t)-\frac{1}{b-a} \int_{a}^{b} f\left((1-t)\left(\frac{b-x}{2}\right)\right) d x .
$$

Proof. Since for $p(x)=\frac{1}{b-a}, x \in[a, b], S_{p}(t)=L_{p}(t)=L(t)$, for all $t \in[a, b]$. Therefore the proof of the crollary follows directly from the above theorem.

\section{Inequalities for differentiable superquadratic functions}

In this section we give results when $f$ is a differentiable superquadratic function. Those results give refinements of (1.4) and (1.5) in Theorem 1 and refine (1.7) of Theorem 2 when superquadratic function $f$ is nonnegative and hence convex. Here we quote very important result which will be helpful in the sequel of the paper. 
Theorem 1. [14, Theorem 10, p. 5] Let $f$ be superquadratic integrable function on $[0, b]$ and $p(x)$ be non-negative integrable and symmetric about $x=\frac{a+b}{2}, 0 \leq a<b$. Let $I$ be defined as above and let $f p$ be integrable on $[a, b]$, then for $0 \leq s \leq t \leq 1, t>0$, we have the following inequality:

$$
\begin{aligned}
I(s) & \leq I(t)-\int_{a}^{b} \frac{t+s}{2 t} f\left(\left(\frac{t-s}{2}\right)(b-x)\right) p(x) d x \\
& -\int_{a}^{b} \frac{t-s}{2 t} f\left(\left(\frac{t+s}{2}\right)(b-x)\right) p(x) d x .
\end{aligned}
$$

Now we state and prove the first result of this section.

Theorem 2. Let $f$ be superquadratic function on $[0, b]$ and $p(x)$ be nonnegative integrable and symmetric about $x=\frac{a+b}{2}, 0 \leq a<b$. Let $f$ be differentiable on $[a, b]$ such that $f(0)=f^{\prime}(0)=0$ and $p$ is bounded on $[a, b]$, then the following inequalities hold for all $t \in[0,1]$ :

$$
\begin{gathered}
\int_{a}^{b} \frac{1}{2}\left[f\left(\frac{x+a}{2}\right)+f\left(\frac{x+b}{2}\right)\right] p(x) d x-I(t) \\
\leq(1-t)\left[\frac{f(a)+f(b)}{2}(b-a)-\int_{a}^{b} f(x) d x\right]\|p\|_{\infty} \\
-\int_{a}^{b} f\left((1-t)\left(\frac{b-x}{2}\right)\right) p(x) d x,
\end{gathered}
$$

where $\|p\|_{\infty}=\sup _{x \in[a, b]}|p(x)|$ and

$$
\begin{aligned}
& \frac{f(a)+f(b)}{2} \int_{a}^{b} p(x) d x-I(t) \leq\left[\frac{\left(f^{\prime}(b)-f^{\prime}(a)\right)(b-a)}{4}\right. \\
& \left.-f\left(\left|\frac{a-b}{2}\right|\right)\right] \int_{a}^{b} p(x) d x-\int_{a}^{b} f\left(t\left(\frac{b-x}{2}\right)\right) p(x) d x .
\end{aligned}
$$

Proof. By integration by parts, we have that

$$
\int_{a}^{\frac{a+b}{2}}\left(\frac{a+b}{2}-x\right)\left[f^{\prime}(a+b-x)-f^{\prime}(x)\right] d x=\int_{a}^{b}\left(x-\frac{a+b}{2}\right) f^{\prime}(x) d x
$$




$$
=\frac{f(a)+f(b)}{2}(b-a)-\int_{a}^{b} f(x) d x .
$$

Using the substitution rules for integration, under the assumptions on $p$, we have

$$
\begin{gathered}
\int_{a}^{b} \frac{1}{2}\left[f\left(\frac{x+a}{2}\right)+f\left(\frac{x+b}{2}\right)\right] p(x) d x=\int_{a}^{b} \frac{1}{2}\left[f\left(\frac{x+a}{2}\right)\right. \\
\left.+f\left(\frac{a+2 b-x}{2}\right)\right] p(x) d x \\
=\int_{a}^{\frac{a+b}{2}}[f(x)+f(a+b-x)] p(2 x-a) d x
\end{gathered}
$$

and

$$
\begin{gathered}
I(t)=\int_{a}^{b} \frac{1}{2}\left[f\left(t \frac{x+a}{2}+(1-t) \frac{a+b}{2}\right)\right. \\
\left.+f\left(t \frac{a+2 b-x}{2}+(1-t) \frac{a+b}{2}\right)\right] p(x) d x \\
=\int_{a}^{\frac{a+b}{2}}\left[f\left(t x+(1-t) \frac{a+b}{2}\right)\right. \\
\left.+f\left(t(a+b-x)+(1-t) \frac{a+b}{2}\right)\right] p(2 x-a) d x
\end{gathered}
$$

for all $t \in[0,1]$.

Now by the assumptions on $f$, we have that

$$
\begin{gathered}
{\left[f(x)-f\left(t x+(1-t) \frac{a+b}{2}\right)\right] p(2 x-a)+[f(a+b-x)} \\
\left.-f\left(t(a+b-x)+(1-t) \frac{a+b}{2}\right)\right] p(2 x-a) \\
\leq(1-t)\left(\frac{a+b}{2}-x\right)\left[f^{\prime}(a+b-x)-f^{\prime}(x)\right] p(2 x-a) \\
-2 f\left((1-t)\left(\frac{a+b}{2}-x\right)\right) p(2 x-a)
\end{gathered}
$$




$$
\begin{gathered}
\leq(1-t)\left(\frac{a+b}{2}-x\right)\left[f^{\prime}(a+b-x)-f^{\prime}(x)\right]\|p\|_{\infty} \\
-2 f\left((1-t)\left(\frac{a+b}{2}-x\right)\right) p(2 x-a),
\end{gathered}
$$

for all $t \in[0,1]$ and $x \in\left[a, \frac{a+b}{2}\right]$.

From (3.4), (3.5), (3.6) and (3.7) and by the change of variable $x \rightarrow \frac{a+x}{2}$, we get (3.2).

By the assumptions on $f$ and from Lemma 3 , we get that

$$
\frac{f(a)-f\left(\frac{a+b}{2}\right)}{2} \leq \frac{a-b}{4} f^{\prime}(a)-\frac{1}{2} f\left(\left|\frac{a-b}{2}\right|\right)
$$

and

$$
\frac{f(b)-f\left(\frac{a+b}{2}\right)}{2} \leq \frac{b-a}{4} f^{\prime}(b)-\frac{1}{2} f\left(\left|\frac{a-b}{2}\right|\right) .
$$

Adding these inequalities we get that

$$
\frac{f(a)+f(b)}{2}-f\left(\frac{a+b}{2}\right) \leq \frac{\left(f^{\prime}(b)-f^{\prime}(a)\right)(b-a)}{4}-f\left(\left|\frac{a-b}{2}\right|\right) .
$$

Thus

$$
\begin{aligned}
& \frac{f(a)+f(b)}{2} \int_{a}^{b} p(x) d x-f\left(\frac{a+b}{2}\right) \int_{a}^{b} p(x) d x \\
\leq & {\left[\frac{\left(f^{\prime}(a)-f^{\prime}(b)\right)(b-a)}{4}-f\left(\left|\frac{a-b}{2}\right|\right)\right] \int_{a}^{b} p(x) d x . }
\end{aligned}
$$

From (3.1), for $s=0$, we have

$$
f\left(\frac{a+b}{2}\right) \int_{a}^{b} p(x) d x \leq I(t)-\int_{a}^{b} f\left(t\left(\frac{b-x}{2}\right)\right) p(x) d x,
$$

for all $t \in[0,1]$.

From (3.8) and (3.9), we get (3.3). This completes the proof of the theorem.

Remark 3. The inequalities (3.2) and (3.3) refine the inequalities (1.4) and (1.5) in Theorem 1, when the superquadratic function $f$ is non-negative and therefore convex. 
Corollary 4. Let $f$ be superquadratic function on $[0, b]$ and differentiable on $[a, b]$ such that $f(0)=f^{\prime}(0)=0$. If $p(x)=\frac{1}{b-a}$, then we have the following inequalities:

$$
\begin{gathered}
\int_{a}^{b} \frac{1}{2(b-a)}\left[f\left(\frac{x+a}{2}\right)+f\left(\frac{x+b}{2}\right)\right] d x-H(t) \\
\leq \frac{1-t}{b-a}\left[\frac{f(a)+f(b)}{2}(b-a)-\int_{a}^{b} f(x) d x\right] \\
\quad-\frac{1}{b-a} \int_{a}^{b} f\left((1-t)\left(\frac{b-x}{2}\right)\right) d x
\end{gathered}
$$

and

$$
\begin{gathered}
\frac{f(a)+f(b)}{2}-H(t) \leq \frac{\left(f^{\prime}(b)-f^{\prime}(a)\right)(b-a)}{4}-f\left(\left|\frac{a-b}{2}\right|\right) d x \\
-\frac{1}{b-a} \int_{a}^{b} f\left(t\left(\frac{b-x}{2}\right)\right) d x
\end{gathered}
$$

for all $t \in[0,1]$.

Now we give our last result and summarize the results related to it in the remark followed by Theorem 12 .

Theorem 5. Let $f$ be superquadratic function on $[0, b]$ and $p(x)$ be nonnegative integrable and symmetric about $x=\frac{a+b}{2}, 0 \leq a<b$. Let $f$ be differentiable on $[a, b]$ such that $f(0)=f^{\prime}(0)=0$ and $p$ is bounded on $[a, b]$, then for all $t \in[0,1]$ we have the inequality:

$$
\begin{gathered}
I(t)-f\left(\frac{a+b}{2}\right) \int_{a}^{b} p(x) d x \leq(b-a)[G(t)-H(t)]\|p\|_{\infty} \\
-\int_{a}^{b} f\left(\frac{t}{2}(b-x)\right) p(x) d x,
\end{gathered}
$$

where $\|p\|_{\infty}=\sup _{x \in[a, b]}|p(x)|$. 
Proof. By integration by parts, we observe that

$$
\begin{gathered}
t \int_{a}^{\frac{a+b}{2}}\left[\left(x-\frac{a+b}{2}\right) f^{\prime}\left(t x+(1-t) \frac{a+b}{2}\right)\right. \\
\left.+\left(\frac{a+b}{2}-x\right) f^{\prime}\left(t(a+b-x)+(1-t) \frac{a+b}{2}\right)\right] d x \\
=t \int_{a}^{b}\left(x-\frac{a+b}{2}\right) f^{\prime}\left(t x+(1-t) \frac{a+b}{2}\right) d x \\
=(b-a) G(t)-H(t),
\end{gathered}
$$

hold for sll $t \in[0,1]$.

Under the assumptions on $f$, we have that

$$
\begin{gathered}
{\left[f\left(t x+(1-t) \frac{a+b}{2}\right)-f\left(\frac{a+b}{2}\right)\right] p(2 x-a)} \\
+\left[f\left(t(a+b-x)+(1-t) \frac{a+b}{2}\right)-f\left(\frac{a+b}{2}\right)\right] p(2 x-a) \\
\leq t\left(x-\frac{a+b}{2}\right) f^{\prime}\left(t x+(1-t) \frac{a+b}{2}\right) p(2 x-a) \\
+t\left(\frac{a+b}{2}-x\right) f^{\prime}\left(t(a+b-x)+(1-t) \frac{a+b}{2}\right) p(2 x-a) \\
=t\left(\frac{a+b}{2}-x\right)\left[f^{\prime}\left(t(a+b-x)+(1-t) \frac{a+b}{2}\right)\right. \\
\left.-f^{\prime}\left(t x+(1-t) \frac{a+b}{2}\right)\right] p(2 x-a)-2 f\left(t\left|\frac{a+b}{2}-x\right|\right) p(2 x-a) \\
\leq t\left(\frac{a+b}{2}-x\right)\left[f^{\prime}\left(t(a+b-x)+(1-t) \frac{a+b}{2}\right)\right.
\end{gathered}
$$




$$
\left.-f^{\prime}\left(t x+(1-t) \frac{a+b}{2}\right)\right]\|p\|_{\infty}-2 f\left(t\left|\frac{a+b}{2}-x\right|\right) p(2 x-a)
$$

hold for all $t \in[0,1]$ and $x \in\left[a, \frac{a+b}{2}\right]$.

Integrating (3.14) over $x$ on $\left[a, \frac{a+b}{2}\right]$, using (3.13), by the change of variable $x \rightarrow \frac{x+a}{2}$, under the assumptions on $p$, we get (3.12). This completes the proof of the theorem.

Remark 6. The result of Theorem 12 refines (1.7) of Theorem 2, when superquadratic function $f$ is non-negative and therefore convex.

Corollary 7. Let $f$ be superquadratic function on $[0, b]$. Let $f$ be differentiable on $[a, b]$ such that $f(0)=f^{\prime}(0)=0$. If $p(x)=\frac{1}{b-a}, x \in[a, b]$, then

$$
H(t)-f\left(\frac{a+b}{2}\right) \leq[G(t)-H(t)]-\frac{1}{b-a} \int_{a}^{b} f\left(t\left(\frac{b-x}{2}\right)\right) d x
$$

for all $t \in[0,1]$.

\section{References}

[1] S. Abramovich, S. Banić, M. Matić, J. Pečarić, Jensen-Steffensen's and related inequalities for superquadratic functions, Math. Ineq. Appl., 11, pp. 23-41, (2008).

[2] S. Abramovich, J. Barić, J. Pečarić, Fejér and Hermite-Hadamard type inequalities for superquadratic functions, Math. J. Anal. Appl., 344, pp. 1048-1056, (2008).

[3] S. Abramovich, G. Jameson, G. Sinnamon, Refining Jensen's inequality, Bull. Math. Soc. Sci. Math. Roumanie (N.S.) 47 (95), pp. 3-14, (2004). 
[4] S. Banić, J. Pečarić, S. Varošanec, Superquadratic functions and refinements of some classical inequalities, J. Korean Math. Soc. 45, pp. $513-525,(2008)$.

[5] S. Banić, Superquadratic functions, PhD thesis, Zagreb (in Croatian), (2007).

[6] S. S. Dragomir, Two mappings in connection to Hadamard's inequalities, J. Math. Anal. Appl., 167, pp. 49-56, (1992).

[7] S. S. Dragomir, Further properties of some mappings associated with Hermite-Hadamard inequalities, Tamkang. J. Math., 34 (1), pp. 45-57, (2003).

[8] S. S. Dragomir, Y.J. Cho and S.S. Kim, Inequalities of Hadamard's type for Lipschitzian mappings and their applications, J. Math. Anal. Appl., 245, pp. 489-501, (2000).

[9] S. S. Dragomir, D.S. Milošević and J. Sándor, On some refinements of Hadamard's inequalities and applications, Univ. Belgrad. Publ. Elek. Fak. Sci. Math., 4, pp. 3-10, (1993).

[10] L. Fejér, Über die Fourierreihen, II, Math. Naturwiss. Anz Ungar. Akad. Wiss., 24, pp. 369-390, (1906). (In Hungarian).

[11] Ming-In Ho, Fejer inequalities for Wright-convex functions, JIPAM. J. Inequal. Pure Appl. Math. 8 (1), (2007), article 9.

[12] J. Hadamard, Étude sur les propriétés des fonctions entières en particulier d'une function considérée par Riemann J. Math. Pures and Appl., 58, pp. 171-215, (1983).

[13] M. A. Latif, On some refinements of Fejér type inequalities via superquadratic functions.(to appear)

[14] M. A. Latif, On some new Fejér-type inequalities for superquadratic functions. (to appear)

[15] K. L. Tseng, S. R. Hwang and S.S. Dragomir, On some new inequalities of Hermite-Hadamard- Fejér type involving convex functions, Demonstratio Math., XL (1), pp. 51-64, (2007).

[16] K. L. Tseng, S. R. Hwang and S.S. Dragomir, Fejér-type Inequalities (I), (Submitted) Preprint RGMIA Res. Rep. Coll. 12 (2009), No.4, Article 5. [Online http://www.staff.vu.edu.au/RGMIA/v12n4.asp.]. 
[17] K. L. Tseng, S.R. Hwang and S.S. Dragomir, Fejér-type Inequalities (II), (Submitted) Preprint RGMIA Res. Rep. Coll. 12 (2009), Supplement, Article 16, pp.1-12. [Online http://www.staff.vu.edu.au/RGMIA/v12(E).asp.].

[18] K. L. Tseng, S. R. Hwang and S.S. Dragomir, Some companions of Fejér's inequality for convex functions, (Submitted) Preprint RGMIA Res. Rep. Coll. 12 (2009), Supplement, Article 19, pp.1-12. [Online http://www.staff.vu.edu.au/RGMIA/v12(E).asp.].

[19] G.S. Yang and K.L. Tseng, Inequalities of Hermite-Hadamard-Fejér type for convex functions and Lipschitzian functions, Taiwanese $J$. Math., 7(3), pp. 433-440, (2003).

\section{A. Latif}

Department of Mathematics, University of Hail,

Hail 2440,

Saudi Arabia

e-mail : m_amer_latif@hotmail.com 